\title{
Crecimiento somático y relación ARN/ADN en estadios juveniles de Eucinostomus argenteus (Pisces: Gerreidae) en dos localidades del Caribe de Venezuela
}

\author{
Ana Teresa Herrera-Reveles ${ }^{1,3}$, Mairin Lemus ${ }^{2} \&$ Baumar Marín ${ }^{3}$ \\ 1. Postgrado en Ecología, Instituto de Zoología y Ecología Tropical, Facultad de Ciencias, Universidad Central de \\ Venezuela. Apartado 47058, Caracas 1041-A Venezuela. (0058-212) 6051300; anate_herrera@yahoo.com \\ 2. Laboratorio de Ecofisiología, Instituto Oceanográfico de Venezuela, Universidad de Oriente Núcleo Cumaná. \\ Apartado 245, Cumaná, Venezuela. (0058-293) 4002249; mlemus@sucre.udo.ve / mlemus88@ gmail.com \\ 3. Laboratorio de Zooplancton, Instituto Oceanográfico de Venezuela, Universidad de Oriente Núcleo Cumaná. Apartado \\ 245, Cumaná, Venezuela. (0058-293) 4002169; bmarin@sucre.udo.edu.ve / bmarin@ gmail.com
}

Recibido 15-VII-2011. Corregido 14-XI-2011. Aceptado 20-XII-2011.

\begin{abstract}
Somatic growth and RNA/DNA rate of Eucinostomus argenteus (Pisces: Gerreidae) juveniles stages at two localities of the Venezuelan Caribbean. In order to evaluate the association among growth indices of marine fishes at early life stages, the somatic growth rate and physiological conditions of Eucinostomus argenteus were estimated at two Venezuelan North-East zones: Mochima Bay and Cariaco Gulf. The age and somatic growth rate were estimated based on daily growth increments in sagitta otoliths. The physiological conditions were evaluated with proteins concentrations and RNA/DNA rate, which were estimated by spectrofluorometric and fluorometric techniques, respectively, on muscle tissue. Juvenile standard length ranged from 9.80 to $39.20 \mathrm{~mm}$ from 21 to 73 days of age. At all the study localities there were significant and positive correlations between age, otolith diameter and body size, and fitted to a linear regression model. The values of recent growth rate ranged from 0.178 to $0.418 \mathrm{~mm} \mathrm{day}^{-1}$, backcalculated growth rate oscillated between $0.295-0.393 \mathrm{~mm} \mathrm{day}^{-1}$, and RNA/DNA rate ranged from 1.65 to 6.97. Differences were not found between study zones, but there were differences between localities. Despite the fact that there was no correlation between juvenile's somatic growth and RNA/DNA rates, the reported values suggesting a E. argenteus juvenile's positive growth in their natural habitat at localities studied. Nevertheless, in some localities values that indicate poor nutritional conditions were registered, which could affect other future demographic rates as survivor and fecundity. Rev. Biol. Trop. 60 (Suppl. 1): 151-163. Epub 2012 March 01.
\end{abstract}

Key words: Gerreidae, growth rate, nutritional condition, otoliths, RNA/DNA.

Estudios sobre la edad y tasa de crecimiento de las especies de peces en sus estadios tempranos permiten entender procesos vitales en la persistencia de las poblaciones, entre los que se pueden mencionar la mortalidad e identificación de cohortes. Al mismo tiempo, las tasas de crecimiento de los individuos se encuentran directamente relacionadas a la dinámica poblacional a través de su influencia a las tasas de supervivencia, madurez y fecundidad (Jones 2002).
El empleo de diversas metodologías para la estimación del crecimiento de los peces puede generar el historial completo de crecimiento de los individuos e incluso relacionarlo con las condiciones ambientales (Stevenson \& Campana 1992, Clemmesen \& Doan 1996, Gilliers et al. 2006). Actualmente dos de las herramientas que han sido ampliamente empleadas para la evaluación la condición y crecimiento de los peces es el estudio de la micro-estructura de los otolitos y los índices bioquímicos. 
La interpretación de la relación entre la longitud del cuerpo de los peces y del tamaño de su otolito, así como también el análisis de los incrementos diarios y/o anuales de crecimiento en los otolitos, se ha empleado desde los años 70 para estimar tallas y edades, así como para revelar diversos procesos ecológicos y oceanográficos (Panella 1971, Stevenson \& Campana 1992, Sponaugle 2010). Específicamente, a través de análisis de retrocálculo se puede reconstruir la historia de crecimientos de un pez (Secor \& Dean 1992, Stevenson \& Campana 1992)

Por su parte, la relación ARN/ADN genera un índice del metabolismo celular y ha sido empleado como una medida de la tasa de crecimiento y condición nutricional de los individuos (Buckley 1984). Esto se debe a que las concentraciones de ARN varían ampliamente dependiendo de la tasa metabólica del tejido y de la condición fisiológica del organismo, mientras que las concentraciones de ADN tienden a ser constantes (Leslie 1955).

Con la finalidad de evaluar la asociación de índices de crecimiento en estadios tempranos de peces marinos a las condiciones ambientales en las que se encuentran, se estimó la tasa de crecimiento somático y las condiciones fisiológicas de una especie común en las costas del oriente venezolano (Eucinostomus argenteus) en dos zonas con diferentes condiciones ambientales en cuanto a extensión, exposición de corrientes y productividad.

\section{MATERIALES Y MÉTODOS}

Recolección de peces: la mojarra $E$. argenteus fue empleada como organismo modelo de estudio, ya que son individuos comunes en los litorales arenosos y frecuentemente es una especie dominante (Cervigón 1993). Aunque no es una especie de importancia comercial, juega un papel fundamental en la cadena trófica en las costas venezolanas, siendo un enlace importante entre el bentos y grandes depredadores pelágicos (Randall 1967, Rivas et al. 1999).

Considerando la importancia ecológica de los ecosistemas marino-costeros de la región oriental de Venezuela (Martín 1995, Miloslavich et al. 2003), se seleccionaron dos zonas de estudio: la Bahía de Mochima, Parque

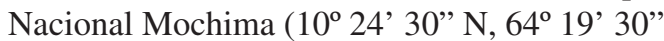
W) y el Golfo de Cariaco $\left(10^{\circ} 31^{\prime} 00^{\prime \prime} \mathrm{N}, 64^{\circ}\right.$ 02'96” W). La Bahía de Mochima se considera una zona de alta productividad relativamente cerrada a corrientes externas, mientras que el Golfo de Cariaco es una zona más extensa, con mayor circulación y entrada de corriente externas, aunque con menores valores de productividad (Okuda et al. 1968, 1978, Expósito 1997, Marín et al. 2004, Quintero et al. 2004).

Dentro de la Bahía de Mochima, los ejemplares de estudio fueron recolectados en dos ensenadas internas: Ensenada de Reyes $\left(10^{\circ} 20^{\prime} 22,473 \prime \prime \mathrm{N}\right.$, y $64^{\circ} 21^{\prime} 42,99^{\prime \prime}$ W) y Ensenada Mochimita $\left(10^{\circ} 20^{\prime} 33,42^{\prime \prime} \mathrm{N}\right.$, y $64^{\circ}$ 21 '04,99" W). En el caso del Golfo de Cariaco, la recolecta de ejemplares se realizó en la Ensenada de Turpialito $\left(10^{\circ} 26^{\prime} 56^{\prime \prime} \mathrm{N}, \mathrm{y}\right.$ $\left.64^{\circ} 02^{\prime} 00^{\prime \prime} \mathrm{W}\right)$. Estas localidades se caracterizan por litorales de arena fina $(250 \mu \mathrm{m})$, con bajas concentraciones de materia orgánica (datos aún no publicados suministrados por el Laboratorio de Ecofisiología, IOV-UDO), con presencia de raíces sumergidas de mangle y praderas de fanerógamas.

Las capturas fueron realizadas en noviembre 2009 y para ello se empleó un chinchorro de arrastre con abertura de malla de 500um. Los ejemplares fueron colocados en hielo y transportados al laboratorio donde se mantuvieron congelados hasta el momento de la disección.

Edad y tasa de crecimiento somático: estas estimaciones fueron realizadas por medio del análisis de los otolitos de los ejemplares capturados y su relación con su morfometría. Para ello, a cada ejemplar se le calculó su peso (P) y se les realizaron mediciones de: longitud total (L.T.) y longitud estándar (L.E.). Posteriormente, se realizó una incisión sobre la cápsula ótica de cada ejemplar, con la finalidad de extraer los otolitos sagitta del lado derecho del individuo, siguiendo los pasos de ruptura, arrastre y aislamiento sobre una placa portaobjeto (Secor et al. 1992). 
Los otolitos fueron colocados sobre una placa de resina sintética termoplástica transparente (Crystalbond ${ }^{\mathrm{TM}}$ ) previamente calentada $\left(>100{ }^{\circ} \mathrm{C}\right)$ en un portaobjeto, y posteriormente se siguió la metodología de raspado propuesta por Secor et al. (1992) para realizar conteo, medición y visualización de los anillos desde el núcleo hasta el borde del otolito. Seguidamente, a través de un programa de procesamiento de imágenes (Sigma Scan Pro 5.0.0), se midió el diámetro del otolito $(\mathrm{Ot})$, se realizó el conteo del número de anillos del otolito y la medición del grosor de cada uno estos anillos, así como también del núcleo del otolito.

A pesar de que en este estudio no se realizó la validación de la formación de los incrementos diarios en los otolitos, se trabajó bajo la hipótesis de que cada banda o anillo representa un día de vida del individuo, considerando que la mayoría de las especies de peces marinos presentan una frecuencia de formación diaria (Campana 1992). De esta forma, la edad de los individuos se estableció según el número de bandas contadas en los otolitos. Por otra parte, el análisis de las variaciones de los anchos de cada anillo permitió discernir las bandas pertenecientes a los períodos de larva e inicio del estadio juvenil, y por ende hacer una estimación aproximada de los períodos de tiempo larvarios de la especie en estudio (Brothers et al. 1983)

La tasa de crecimiento reciente de los individuos, C. Rec ( $\left.\mathrm{mm} \mathrm{día}^{-1}\right)$, fue estimada al relacionar la L.E. de los individuos con la edad (E) en días. De esta forma el valor de la tasa de crecimiento de los individuos corresponde a la pendiente del modelo de crecimiento que mejor ajuste (Thorrold \& Williams 1989, Neuman et al. 2001). La tasa de crecimiento retrocalculada (C. Retro) se obtuvo utilizando las tallas pretéritas (Thorrold \& Williams 1989), considerando que el tamaño del otolito es proporcional al tamaño del individuo. Para el cálculo de dichas tallas pretéritas se empleó el ajuste al relacionar el diámetro del otolito con el tamaño del pez. Así, se empleó la ecuación resultante para determinar las longitudes pretéritas a cada edad, cuya pendiente representó la tasa pretérita media de crecimiento para cada día de la vida del ejemplar.

Condición nutricional: a cada ejemplar se le retiró la cabeza, abdomen y aletas, para utilizar el tejido restante en los análisis de concentraciones de proteínas, ADN y ARN. Cada tejido fue colocado en $100 \mu$ de solución de sarcosina (N-lauroy-sarcosina) $1 \%$ preparada con buffer TRIS-EDTA (5.0mmol TRIS-HCl; 0.5mmol EDTA; pH 7.5) y se homogeneizó, al momento de colocarle la sarcosina, a los 30 minutos y a los 60 minutos posteriores. Seguidamente, se agregaron $900 \mu \mathrm{l}$ de buffer y se centrifugó a $2500 \mathrm{rpm}$ por 15 minutos, obteniéndose así para cada individuo una solución de $1 \mathrm{ml}$. Todo lo mencionado anteriormente se preparó manteniendo el tejido frío. Del sobrenadante se tomaron muestras por duplicado para la cuantificación de ARN y ADN utilizando la metodología de Clemmesen (1993). Las proteínas totales fueron determinadas mediante la metodología descrita por Bradford (1976).

Análisis Estadísticos: se realizaron análisis de correlación y regresión (Sokal \& Rohlf 1995), para establecer relaciones entre las variables medidas en cada juvenil para cada localidad: L.T., L.E., P, Ot, E, proteínas, ADN, ARN y ARN/ADN. Igualmente, se realizaron correlaciones y regresiones entre C. Retro y el índice ARN/ADN por localidad. Todo esto revisando previamente la normalidad y homogeneidad de varianza.

Las distintas tasas de crecimiento se compararon a través de un análisis de covarianza (Sokal \& Rohlf 1995). Comparaciones entre localidades fueron realizadas a través de un ANOVA de una vía, para detectar diferencias entre las concentraciones de proteínas, índice ARN/ADN y la tasa de crecimiento retrocalculada, revisando previamente la normalidad y homogeneidad de varianza. Posteriormente, se realizó un test de Tukey para discernir las localidades que mostraban diferencias con un nivel de significancia de 0.05. En el caso de la evaluación del cambio de ancho de las bandas diarias, debido a la naturaleza de los datos, 
se realizó la comparación del promedio del ancho de estos incrementos diarios por localidad a través de una prueba Kruskal-Wallis. Estos análisis fueron realizados a través del programa SPSS 16.0.

Para una mejor visualización de las posibles diferencias entre localidades se realizó una ordenación no métrico de escalas multidimensionales (MDS, PRIMER 5), basado en el índice de distancia euclideana (Clarke 1993). Seguidamente, para establecer la significancia del MDS se realizó un análisis de similitud (ANOSIM, PRIMER 5), el cual es un método de permutación aleatoria en la matriz de distancias. El intervalo de similitud global $R$ es una medida comparativa del grado de separación entre grupo $(0 \leq R \leq 1)$, de forma tal que cuando $R$ se aproxima a cero no existe separación entre grupos (Clarke 1993).

\section{RESULTADOS}

Los caracteres morfométricos para los juveniles de E. argenteus presentan intervalos similares en las tres localidades de estudio, así como también la edad y el peso de los individuos (Cuadro 1). En cada una de las localidades de estudio, los análisis de regresión y correlación de las variables morfométricas con el tamaño del otolito, el peso y la edad de los juveniles se ajustaron a un modelo lineal y fueron significativos $(\mathrm{p}<0.001)($ Cuadro 2).

\section{Tasa de crecimiento reciente}

La edad fue un parámetro relacionado con la longitud estándar en las tres localidades (Cuadro 2). De esta forma, con esta relación se determinó la tasa de crecimiento reciente de las tallas en los estadios tempranos de $E$. argenteus $\left(\mathrm{mm} \mathrm{día}^{-1}\right)$, la cual es diferente entre localidades (ANCOVA, $\mathrm{F}=20.16, \mathrm{p}<0.0001$ ) encontrándose los valores mayores dentro de la Bahía de Mochima (Cuadro 3).

Igualmente, en las tres localidades se observó correlación entre la edad y el peso de los individuos (Cuadro 2), y por ende se estimó la tasa de crecimiento en peso para los estadios tempranos de la especie. Los valores de crecimiento se registraron entre 0.003 y $0,013 \mathrm{~g} \mathrm{día}^{-1}$, sin encontrarse diferencias entre localidades (ANCOVA, $\mathrm{F}=1.53, \mathrm{p}=0.2214$ ).

\section{Tasa de crecimiento retrocalculado}

Debido a la proporcionalidad encontrada entre la longitud estándar de los ejemplares y el diámetro del otolito, así como la correlación existente entre la edad y la longitud estándar, se pudo modelar el crecimiento retrocalculado en cada una de las localidades evaluadas (Cuadro 3). De esta forma, se estimó el valor menor dentro de la Ensenada de Reyes, una de las localidades dentro de la Bahía de Mochima (ANOVA, $\mathrm{F}=8.415, \mathrm{p}=0.001$ ).

CUADRO 1

Variables morfométricas, peso y edad de E. argenteus

TABLE 1

Morophometrics variables, weight and age of E. argenteus

\begin{tabular}{cccc} 
Variable & E. Reyes $($ Bahía Mochima) & E. Mochimita (Bahía Mochima) & Turpialito (Golfo Cariaco) \\
L.T. & $20.520 \pm 7.910(\mathrm{n}=33)$ & $22.05 \pm 7.122(\mathrm{n}=47)$ & $21.573 \pm 8.638(\mathrm{n}=46)$ \\
L.E. & $16.045 \pm 6.073(\mathrm{n}=33)$ & $18.757 \pm 5.947(\mathrm{n}=47)$ & $17.708 \pm 6.909(\mathrm{n}=46)$ \\
Ot & $0.883 \pm 0.498(\mathrm{n}=26)$ & $0.875 \pm 0.270(\mathrm{n}=31)$ & $0.999 \pm 0.341(\mathrm{n}=41)$ \\
P & $0.174 \pm 0.296(\mathrm{n}=32)$ & $0.172 \pm 0.155(\mathrm{n}=47)$ & $0.165 \pm 0.167(\mathrm{n}=46)$ \\
E & $30.250 \pm 9.261(\mathrm{n}=25)$ & $32.650 \pm 6.235(\mathrm{n}=20)$ & $43.520 \pm 13.135(\mathrm{n}=23)$ \\
\hline
\end{tabular}

L.T.: Longitud total (mm), L.E.: Longitud estándar (mm), Ot: diámetro del otolito (mm), P: Peso (g), E: Edad (Días), D.E.: Desviación estándar; n: número de mediciones. 


\section{CUADRO 2}

Relaciones entre la longitud estándar, el diámetro del otolito, la edad y el peso en juveniles de E. argenteus en las localidades de estudio

TABLE 2

Relationships among standard lenght, otoliths diameter, age and weight of E. argenteus juveniles at study localities

\begin{tabular}{|c|c|c|c|c|c|}
\hline Localidad & Relación & Ecuación & $r^{2}$ & $r$ & $\mathrm{n}$ \\
\hline \multirow{4}{*}{$\begin{array}{l}\text { E. Reyes } \\
\text { (Bahía Mochima) }\end{array}$} & L.E. vs Ot & L.E. $=11.856 \mathrm{Ot}+6.472$ & $0.817 * *$ & $0.684 * *$ & 26 \\
\hline & L.E. $v s \mathrm{E}$ & L.E. $=0.4186 \mathrm{E}+2.9363$ & $0.568 * *$ & $0.754 * *$ & 24 \\
\hline & $\mathrm{P} v s \mathrm{Ot}$ & $\mathrm{P}=1.6773 \mathrm{Ot}+0.6333$ & $0.862 * *$ & $0.705^{* *}$ & 26 \\
\hline & $\mathrm{P} v s \mathrm{E}$ & $\mathrm{P}=0.0133 \mathrm{E}-0.319$ & $0.589^{* *}$ & $0.428 * *$ & 24 \\
\hline \multirow{4}{*}{$\begin{array}{l}\text { E. Mochimita } \\
\text { (Bahía Mochima) }\end{array}$} & L.E. $v s \mathrm{Ot}$ & L.E. $=14.145 \mathrm{Ot}+5.8778$ & $0.473 * *$ & $0.643 * *$ & 32 \\
\hline & L.E. vs E & L.E. $=0.3446 \mathrm{E}+4.8002$ & $0.496 * *$ & $0.647 * *$ & 18 \\
\hline & $\mathrm{P} v s \mathrm{Ot}$ & $\mathrm{P}=1.277 \mathrm{Ot}+0.666$ & $0.456^{* *}$ & $0.639 * *$ & 32 \\
\hline & $\mathrm{P} v s \mathrm{E}$ & $\mathrm{P}=0.0063 \mathrm{E}-0.0996$ & $0.302 * *$ & $0.584 * *$ & 20 \\
\hline \multirow{4}{*}{$\begin{array}{l}\text { Turpialito } \\
\text { (Golfo Cariaco) }\end{array}$} & L.E. $v s \mathrm{Ot}$ & L.E. $=18.207 \mathrm{Ot}-0.0011$ & $0.812 * *$ & $0.798 * *$ & 41 \\
\hline & L.E. $v s \mathrm{E}$ & L.E. $=0.178 \mathrm{E}+6.734$ & $0.677 * *$ & $0.620 * *$ & 23 \\
\hline & $\mathrm{P} v s \mathrm{Ot}$ & $\mathrm{P}=1.277 \mathrm{Ot}+0.666$ & $0.823 * *$ & $0.811^{* *}$ & 41 \\
\hline & $\mathrm{P} v s E$ & $\mathrm{P}=0.0038 \mathrm{E}-0.0817$ & $0.660 * *$ & $0.684 * *$ & 23 \\
\hline
\end{tabular}

L.E.: Longitud estándar, Ot: diámetro del otolito, E: Edad, P: Peso. $\mathrm{r}^{2}$ : coeficiente de determinación, r: índice de correlación, $\mathrm{n}$ : número de individuos $* * \mathrm{p}<0.001 ;$ ns: no significativo.

\section{CUADRO 3}

Valores de crecimiento reciente (C. Rec) obtenidos del modelo lineal ajustado a los datos de longitud estándar -edad y peso- edad, y valores promedio y desviación estándar de la tasa de crecimiento retrocalculada (C. Retro), relación ARN/ADN, concentraciones de proteínas de E. argenteus en las tres localidades de estudio

TABLE 3

Values of recent growth (C. Rec) from the lineal model fitted to the data of standard length -age and weightage, and mean values with their standard deviation of backcalculated growth rate (C. Retro), RNA/DNA rate, proteins concentrations of E. argenteus at three study locations

\begin{tabular}{|c|c|c|c|c|c|}
\hline Localidad & $\begin{array}{l}\text { C. Rec } \\
\left(\mathrm{g} \mathrm{día}^{-1}\right)\end{array}$ & $\begin{array}{c}\text { C. Rec } \\
\left(\mathrm{mm} \mathrm{día}^{-1}\right)\end{array}$ & $\begin{array}{l}\text { C. Retro } \\
\left(\mathrm{mm} \mathrm{día}^{-1}\right)\end{array}$ & ARN/ADN & $\begin{array}{c}\text { Proteínas } \\
\left(\mathrm{mg} \mathrm{g}^{-1} \text { tejido húmedo) }\right.\end{array}$ \\
\hline $\begin{array}{l}\text { E. Reyes } \\
\text { (Bahía Mochima) }\end{array}$ & $\begin{array}{c}0.013 \\
(n=24)\end{array}$ & $\begin{array}{c}0.418 \\
(n=24)\end{array}$ & $\begin{array}{c}0.295 \pm 0.050 \\
(\mathrm{n}=24)^{*} * \mathrm{~A}\end{array}$ & $\begin{array}{c}2.46 \pm 1.36 \\
(\mathrm{n}=17)^{\mathrm{X}}\end{array}$ & $\begin{array}{c}25.75 \pm 17.33 \\
\quad(\mathrm{n}=28)^{1}\end{array}$ \\
\hline $\begin{array}{l}\text { E. Mochimita } \\
\text { (Bahía Mochima) }\end{array}$ & $\begin{array}{c}0.006 \\
(n=20)\end{array}$ & $\begin{array}{c}0.334 \\
(\mathrm{n}=18)\end{array}$ & $\begin{array}{c}0.352 \pm 0.080 \\
\quad(n=18)^{B}\end{array}$ & $\begin{array}{l}6.97 \pm 5.68 \\
(\mathrm{n}=11) * * \mathrm{Y}\end{array}$ & $\begin{array}{c}12.56 \pm 5.68 \\
(\mathrm{n}=29) * * 2\end{array}$ \\
\hline $\begin{array}{l}\text { Turpialito } \\
\text { (Golfo Cariaco) }\end{array}$ & $\begin{array}{c}0.003 \\
(n=23)\end{array}$ & $\begin{array}{c}0.178 \\
(n=23)\end{array}$ & $\begin{array}{c}0.393 \pm 0.083 \\
(n=16)^{B}\end{array}$ & $\begin{array}{c}1.65 \pm 2.59 \\
(\mathrm{n}=16)^{\mathrm{X}}\end{array}$ & $\begin{array}{c}21.01 \pm 2.59 \\
(n=29)^{1}\end{array}$ \\
\hline
\end{tabular}

n: número de individuos. Se utilizaron pruebas estadísticas para observar diferencias entre localidades para la tasa de crecimiento retrocalculada (C. Retro), ARN/ADN y concentraciones de proteínas y éstas son marcadas por ** y representadas por diferencias en letras o números (usando A, B; X, Y; 1,2)

\section{Cambios en la microestructura del otolito}

Los otolitos sagitta de los ejemplares evaluados presentaron un núcleo redondeado de 10 micras de diámetro, aproximadamente. Se observó un patrón de bandas similar en las tres localidades, en donde el ancho de las primeras 8-12 bandas presentaron los menores valores $(<5 \mu \mathrm{m})$, posteriormente se registró un aumento gradual en las siguientes 5-10 bandas y finalmente se puede observar pequeñas oscilaciones en los valores del resto de las bandas de crecimiento 
(entre 4 y 8 um, aproximadamente) (Fig. 1 y 2). El ancho de las bandas en los otolitos de los estadios iniciales de E. argenteus mostraron diferencias entre las localidades de estudio, específicamente a partir de la banda 15 , en donde los mayores valores fueron reportados para Turpialito (Kruskal-Wallis, $\mathrm{H}=651.69, \mathrm{p}<0.0001$ )

\section{Condición Nutricional}

El índice de relación ARN y ADN, y las concentraciones de proteínas, presentaron diferencias entre localidades (ANOVA; $\mathrm{F}=$ $48.73 \mathrm{p}=0.0000 ; \mathrm{F}=8.99 \mathrm{P}=0.0007$; respectivamente). Los mayores valores del índice ARN/ADN se encontraron en una de las localidades de la Bahía de Mochima (Ensenada Mochimita) (Tukey Test; $P=0.0000$ ), mientras que los mayores valores de concentración de proteínas estuvieron presenten en la localidad del Golfo de Cariaco (Turpialito) y Ensenada de Reyes (Bahía de Mochima) (Tukey Test; $P=$ 0.0000) (Cuadro 3).

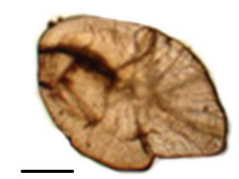

$3 \overline{\mathrm{mm}}$
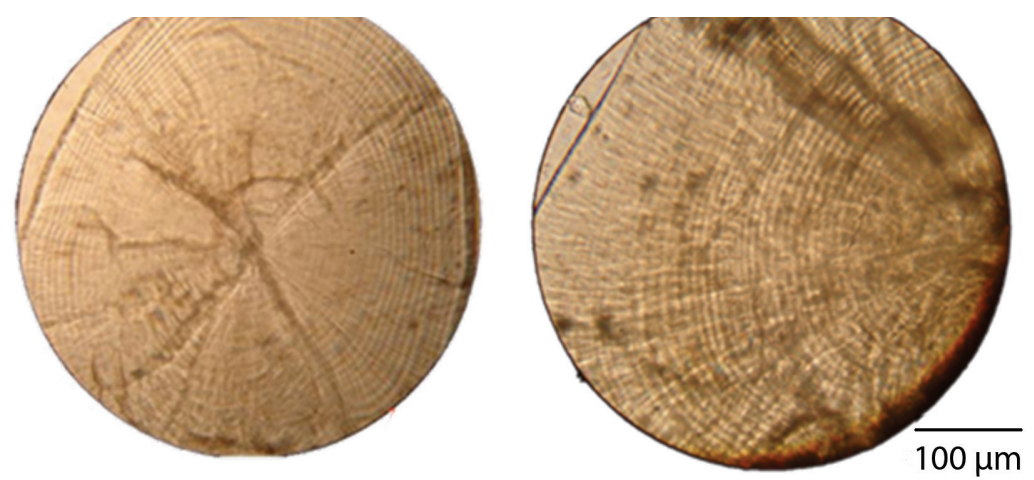

Fig. 1. Otolito sagitta de un ejemplar E. argenteus de $11 \mathrm{~mm}$ de longitud estándar, mostrando el núcleo y 53 líneas de crecimiento.

Fig. 1. Sagitta otolith of a $11 \mathrm{~mm}$ standard length E. argenteus showing the core and 53 growth increments.

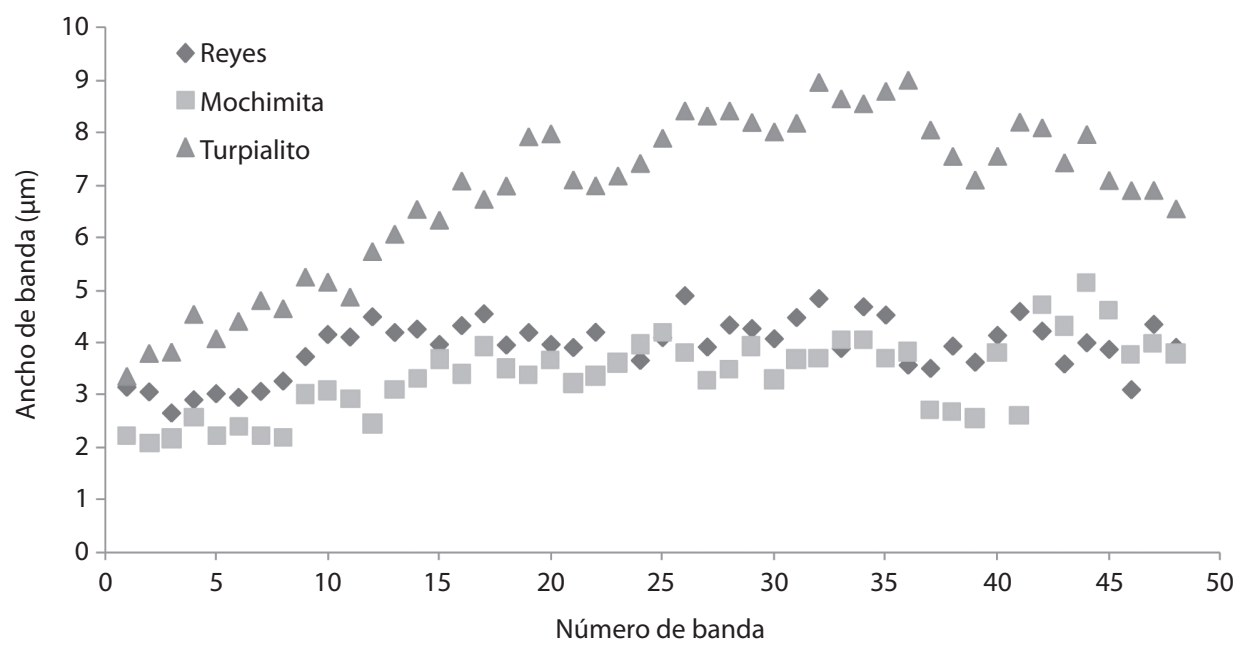

Fig. 2. Variación del ancho promedio de las bandas registradas en los otolitos sagitta de estadios iniciales de E. argenteus. Fig. 2. Variation of the mean increments widths in sagitta otoliths of E. argenteus at early stages. 
Al relacionar las proteínas, ácidos nucléicos y el índice ARN/ADN con ciertas variables morfométricas de los individuos, se observó que en Ensenada de Reyes y Turpialito existen correlaciones significativas entre la concentración de proteínas y peso corporal, así como también entre la concentración de proteínas y longitud estándar, lo cual no se evidencia para Ensenada Mochimita (Cuadro 4). Igualmente, se buscó una relación entre las tasas de crecimiento somático retrocalculado y el índice ARN/ADN; sin embargo, a pesar de parecer adaptarse a un modelo lineal esto no fue significativo para ninguna de las tres localidades de estudio (Cuadro 4).

Un análisis de ordenación, considerando los valores obtenidos de las concentraciones de proteínas, el índice ARN/ADN y la tasa de crecimiento somático retrocalculado, presenta separación de los individuos entre localidades, con un valor de stress de 0.04 (Fig. 3). Esta separación es significativa, ya que la prueba de análisis de similaridades arrojó un $\mathrm{R}$ global igual a 0.467 , con un nivel de significancia igual a 0.01. Específicamente, los resultados

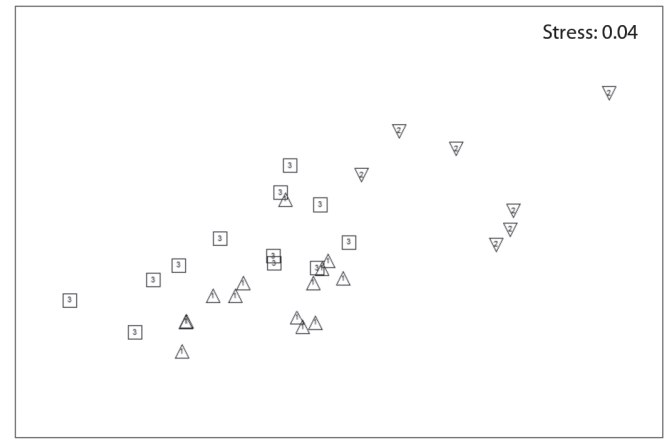

Fig 3. Ordenación multivariada (MDS) de los ejemplares evaluados en las localidades de E. Reyes (1), E. Mochimita (2) y Turpialito (3), empleando los valores de tasa de crecimiento retrocalculado (C. Retro), concentración de proteínas y relación ARN/ADN.

Fig. 3. Metric dimensional scaling (MDS) ordinations of juveniles evaluated from E. Reyes (1), E. Mochimita (2) and Turpialito (3), using the values of backcalculated growth rate (C. Retro), proteins concentrations and RNA/ DNA rates.

indican que la Ensenada Mochimita es altamente diferente a las otras localidades, mientras que Ensenada de Reyes y Turpialito son

\section{CUADRO 4}

Relaciones entre las concentraciones de proteínas, ácidos nucléicos y el índice ARN/ADN con variables morfométricas y la tasa de crecimiento somático retrocalculada en juveniles de E. argenteus en las localidades de estudio

TABLE 4

Relationships among proteins concetrations, nucleics acids, RNA/DNA rates and morphometrics variables, somatic backcalculated growth rate of E. argenteus at study localities

\begin{tabular}{|c|c|c|c|c|c|}
\hline Localidad & Relación & Ecuación & $r^{2}$ & $\mathrm{r}$ & $\mathrm{n}$ \\
\hline \multirow{4}{*}{$\begin{array}{l}\text { E. Reyes } \\
\text { (Bahía Mochima) }\end{array}$} & $\mathrm{P} v s \mathrm{Pr} \diamond$ & $\mathrm{P}=1.9379 \mathrm{Pr}^{-1.217}$ & $0.773 * *$ & $-0.879 * *$ & 29 \\
\hline & L.E $v s \operatorname{Pr}^{\diamond}$ & L.E. $=34.203 \operatorname{Pr}^{-0.246}$ & $0.636 * *$ & $-0.590 * *$ & 29 \\
\hline & $\mathrm{E} v s \operatorname{Pr}^{\diamond}$ & $\mathrm{E}=51.037 \mathrm{Pr}^{-0.16}$ & $0.342 \mathrm{~ns}$ & $-0.392 \mathrm{~ns}$ & 20 \\
\hline & Gr vs ARN:ADN $\diamond 0$ & $\mathrm{Gr}=-0.0731(\mathrm{ARN}: \mathrm{ADN})+1.079$ & $0.457 \mathrm{~ns}$ & $0.473 \mathrm{~ns}$ & 11 \\
\hline \multirow{4}{*}{$\begin{array}{l}\text { E. Mochimita } \\
\text { (Bahía Mochima) }\end{array}$} & $\mathrm{P} v s \operatorname{Pr} \Delta \Delta\rangle$ & $\mathrm{P}=0.1148 \mathrm{e}^{-0.029 \operatorname{Pr}}$ & $0.218 \mathrm{~ns}$ & $-0.426 * *$ & 29 \\
\hline & L.SE. vs $\operatorname{Pr} \Delta 00$ & L.SE. $=17.773 \mathrm{e}^{-0.01 P r}$ & $0.151 \mathrm{~ns}$ & $-0.449 * *$ & 29 \\
\hline & E vs $\operatorname{Pr} \Delta 00$ & $\mathrm{E}=4.525 \mathrm{e}^{-0.004 \operatorname{Pr}}$ & $0.036 \mathrm{~ns}$ & $-0.363 \mathrm{~ns}$ & 20 \\
\hline & Gr vs ARN:ADN ${ }^{\diamond \diamond}$ & $\mathrm{Gr}=1.10^{-5}(\mathrm{ARN} / \mathrm{ADN})+0.038$ & $0.422 \mathrm{~ns}$ & $0.700 \mathrm{~ns}$ & 5 \\
\hline \multirow{4}{*}{$\begin{array}{l}\text { Turpialito } \\
\text { (Golfo Cariaco) }\end{array}$} & $\mathrm{P} v s \operatorname{Pr} \Delta \Delta 0$ & $\mathrm{P}=0.1005 \mathrm{e}^{-0.032 \mathrm{Pr}}$ & $0.511 * *$ & $0.747 * *$ & 29 \\
\hline & L.SE. vs $\operatorname{Pr}{ }^{\diamond}$ & L.SE. $=-0.1502 \operatorname{Pr}+18.167$ & $0.334 * *$ & $-0.665 * *$ & 29 \\
\hline & $\mathrm{E} v s \operatorname{Pr} \diamond 0$ & $E=-0.0027 \operatorname{Pr}+33.36$ & $0.000 \mathrm{~ns}$ & $-0.328 * *$ & 22 \\
\hline & Gr vs ARN:ADN $\diamond$ & $\mathrm{Gr}=0.01(\mathrm{ARN}: \mathrm{ADN})+0.122$ & $0.431 \mathrm{~ns}$ & $0.467 \mathrm{~ns}$ & 10 \\
\hline
\end{tabular}

L.E.: Longitud Estándar, E: Edad, P: Peso, Pr: Proteínas, Gr: tasa de crecimiento retrocalculado, $\mathrm{r}^{2}$ : coeficiente de determinación, r: índice de correlación, n: número de individuos $* * \mathrm{p}<0.001$; ns: no significativo. ${ }^{\vartheta}$ Relación ajustada a un modelo potencial. ${ }^{\diamond}$ Relación ajustada a un modelo lineal ${ }^{\diamond \diamond}$ Relación ajustada a un modelo polinómico. 
bastante similares entre si, con respecto a las variables evaluadas (Cuadro 5).

\section{CUADRO 5}

Análisis de similitud (ANOSIM). Prueba entre localidades de estudio. El R global de similitud fue de 0.467 con nivel de significancia estadística $\mathrm{p}=0.01$

TABLE 5

Analysis of similarities (ANOSIM). Test among study localities. The similitude global $\mathrm{R}$ was 0.467 with statistic significance level $\mathrm{p}=0.01$

\begin{tabular}{lcc}
\multicolumn{1}{c}{ Localidades } & $R$ & $\mathrm{P}$ \\
E. Reyes - E. Mochimita & 0.889 & 0.001 \\
E. Reyes - Turpialito & 0.127 & 0.410 \\
E. Mochimita - Turpialito & 0.700 & 0.001 \\
\hline
\end{tabular}

\section{DISCUSIÓN}

En esta evaluación, se estableció para $E$. argenteus una relación lineal entre la longitud estándar de los individuos capturados y el tamaño de su otolito, lo cual coincide con varias evaluaciones realizadas a otras especies de peces dentro y fuera del Caribe, en donde esto se observa claramente en los primeros estadios de vida (Jones 2002). La existencia de una relación entre la longitud corporal y el tamaño del otolito, aunado a reportes de la validación sobre la relación entre el crecimiento somático y los incrementos diarios en los otolitos, soportan el uso del ancho o amplitud de los incrementos como una medida de la tasa de crecimiento somático (Secor \& Dean 1989, Campana \& Thorrold 2001). De esta forma, se pudo estimar la tasa de crecimiento promedio de E. argenteus en las localidades de estudio, para la cual hasta la fecha no se había evaluado.

Las tasas de crecimiento en peso de $E$. argenteus son relativamente bajos a los valores estimados para otras especies de mojarras. Estudios en laboratorio han estimado que la tasa de crecimiento de la masa corporal de los gerreidos es lento durante sus primeros estadios, tales como Eugerres plumieri para el Caribe $\left(0.06 \mathrm{~g} \mathrm{día}^{-1}\right)$, E. currani $(0.26-0.36$ g día $\left.{ }^{-1}\right)$ y Diapterus peruvians (0.31- $0.45 \mathrm{~g}$ día ${ }^{-1}$ ) para el Pacífico (Tucker \& Jory 1991, Rubio et al. 2004). Por otra parte, en el caso del crecimiento de tallas, diversos estudios en adultos de la dinámica espacio-temporal de ciertas especies de gerreidos, los han identificado como peces de rápido crecimiento. En estos estudios se han evaluado los parámetros de la ecuación de crecimiento de von Bertalanffy, en donde los valores reportados del coeficiente de crecimiento (k) de dicha ecuación se encuentra alrededor de 1, tal es el caso de E. plumieri $(\mathrm{k}=$ $\left.1.27 \mathrm{año}^{-1}\right)$ (Venezuela), Gerres longirostris $(\mathrm{k}=$ 1.1 año $^{-1}$ ) (Golfo de Arabia), Pentaprion longimanus $\left(\mathrm{k}=1.8\right.$ año $^{-1}$ ) (Mar Java) (Sadhotomo 1983, Grandcourt et al. 2006, Montaño 2009).

$\mathrm{Al}$ querer realizar comparaciones con otras especies de la zona de estudio, se tiene que para el área sólo se han hecho estudios similares para el clupeido Sardinella aurita, reportándose valores mayores a E. argenteus dentro del Parque Nacional Mochima (0.66 y $\left.1.10 \mathrm{~mm} \mathrm{día}^{-1}\right)$, mientras que en Turpialito las tasas de crecimiento son similares $\left(0.32 \mathrm{~mm} \mathrm{día}^{-1}\right)$ (Balza \& Marín 2000, Balza et al. 2006, Ramírez \& Marín 2006).

En cuanto al crecimiento de los otolitos, las variaciones diarias encontradas en el ancho de las bandas refleja los cambios en el crecimiento somático de los individuos de E. argenteus. En general, las variaciones en el ancho de las bandas presentes tienden generalmente a aumentar con la edad (Thorrold \& Williams, 1989), y por lo tanto se puede estimar los períodos de las diferentes fases o estadios de los individuos. De esta forma, del patrón de ancho de las bandas de E. argenteus en las localidades de estudio, y siguiendo el patrón propuesto por Brothers \& McFarland (1981), se puede distinguir que por el pequeño grosor de las primeras bandas el período larvario de la especie se encuentra entre 12 y 15 días. Por su parte, a partir de la banda número 16 no se evidencia un patrón que permita estimar cambios de post-larva a pre-juvenil y de pre-juvenil a juvenil, lo cual puede estar asociado a cambios de factores internos o externos del individuo (Wilson \& McCormick 1999). 
Los períodos de las fases mencionadas anteriormente, coinciden con diversas especies marinas, y han sido considerados como períodos relativamente cortos (Swearer et al. 1999, Claro \& Lindeman 2003, Balza \& Marín 2000; Raventós \& Macpherson 2001, Marín 2009). Estos se ajustan a las hipótesis de que crecimientos rápidos y mayores presentan ventajas en la sobrevivencia de los individuos (Houde 2009).

En el caso de los mayores tamaños de las bandas de crecimiento de los otolitos de los individuos de Turpialito con respecto a los de la Bahía de Mochima, pueden deberse a factores exógenos o endógenos particulares que se encuentran afectando el crecimiento de las bandas sin que aparentemente se afecte drásticamente el crecimiento somático, tal cual ha sido observado para otras especies de la región (Ramírez \& Marín 2006).

Igualmente, al considerar los valores de la relación ARN/ADN obtenidos, se puede inferir que los individuos de E. argenteus, de las localidades evaluadas, se encuentran en crecimiento, ya que se ha estimado que el valor límite inferior de ARN/ADN necesario para sobrevivir es 1.0, debido a que individuos que se encuentren en períodos de inanición prolongados presentan decrecimiento en la síntesis de ribosomas e incluso procesos de degradación de los mismos, lo cual indica pérdida de ARN (Alfaro et al. 2002). No obstante, se encuentran diferencias entre localidades en cuanto a su condición nutricional, en donde los individuos de Turpialito y Ensenada de Reyes presentan valores que han sido considerados bajos en otras especies, e incluso en evaluaciones experimentales se ha llegado a concluir que para ciertas especies valores por debajo de 3 indican condiciones nutricionales pobres (Bulow 1987, Richard et al. 1991, Cunha et al. 2003, Caldarone 2005).

Varios estudios de individuos silvestres han encontrados correlación positiva entre el crecimiento de los últimos incrementos de los otolitos y la proporción de ARN/ADN (Clemmesen \& Doan 1996, Balza et al. 2006, Balza et al. 2007). Sin embargo, al igual que en este estudio, hay otros reportes en donde no se ha encontrado correlaciones entre los ácidos nucléicos y el crecimiento de los individuos, sugiriendo que el índice ARN/ADN se encuentra más relacionado a la condición nutricional de los individuos que a su crecimiento (Bergeron 1997, Gilliers et al. 2006, Frommel \& Clemmesen 2009). Esto pudiera ser explicado por medio de evidencias de transiciones de la composición bioquímica de los individuos durante sus cambios ontogénicos y su reorganización celular.

El contenido proteico de los peces tiende a cambiar durante su crecimiento, en donde las larvas requieren grandes cantidades de proteínas para un rápido crecimiento, y estos tienden a decrecer a medida que llegan a la madurez, pudiendo llegar a ser la concentración de lípidos y no las proteínas los responsables del incremento en masa y de tallas de los individuos (Caldarone et al. 2006, Frommel \& Clemmesen 2009). De esta forma, las relaciones entre el crecimiento somático y el índice ARN/ADN puede cambiar dependiendo del estadio que se esté evaluando (Peck et al. 2003, Caldarone 2005). Al mismo tiempo, la explicación mencionada anteriormente, se ve fortalecida con las relaciones negativas encontradas entre las concentraciones de proteínas de los individuos y los pesos, longitudes estándares y edades de los mismos.

Al considerar que las zonas estudiadas presentan masas de aguas, patrones hidrodinámicos y productividad primaria diferente (Okuda et al. 1968, 1978, Expósito 1997, Marín et al. 2004, Quintero et al. 2004, Márquez et al. 2007), los parámetros biológicos evaluados sobre E. argenteus sugieren poco efecto de estas diferencias sobre la tasa de crecimiento y la condición nutricional en dicha especie.

Por otra parte, las diferencias expuestas entre las localidades estudiadas dentro de la Bahía de Mochima, reflejan la posible interferencia de factores locales sobre la tasa de crecimiento y la condición nutricional de los estadios iniciales de E. argenteus. Entre los factores particulares del hábitat que pudieran explicar las diferencias encontradas entre las 
localidades de la Bahía de Mochima, pudieran destacarse la presión de depredadores, temperatura, concentración y composición de presas, turbidez, efecto antropogénico, tal como han sido propuestos para otras regiones (Gibson 1994, Meekan et al. 2003, Gilliers et al. 2006, Sponaugle et al. 2009, Sponaugle et al. 2010). Específicamente al sur de la Bahía de Mochima, y muy cercana a la Ensenada Mochimita, se encuentra un sistema de tratamiento de aguas residuales que descarga directamente al mar, de forma tal que a esta área junto a sus adyacencias se le ha caracterizado como un ambiente mesotrófico - eutrófico por las altas concentraciones de nutrimentos y un elevado crecimiento del plancton (Expósito 1997, Narváez 2011).

Al mismo tiempo, en zonas muy cercanas a la laguna de oxidación se han encontrado diversas concentraciones de ciertos metales (datos aún no publicados, suministrados por el Laboratorio de Ecofisiología, IOV-UDO), lo cual pudiera explicar los valores elevados de la relación ARN/ADN en los individuos de la Ensenada Mochimita, puesto que en caso de exposición de contaminantes, un incremento en las concentraciones de ARN pudiera relacionarse a la inducción del sistema de desintoxicación proteico, tal y como lo reportaron Fonseca et al. (2009) en evaluaciones experimentales sobre juveniles de Solea senegalensis a diferentes concentraciones de cobre. De esta forma los juveniles de E. argenteus pudieran estar presentando una movilización de proteínas musculares destinada a compensar el efecto de factores estresantes que posiblemente estén causando las descargas de la laguna de oxidación cercana.

Evaluaciones futuras deberían incrementar el número de localidades dentro de las zonas de estudio, así como también realizar experimentos de crecimiento y condición nutricional en condiciones controladas que permitan dilucidar los patrones de crecimientos somáticos y del otolito, así como también las condiciones nutricionales de los individuos, bajo diferentes condiciones de temperatura, alimento, contaminantes e incluso por presión de depredadores.

\section{AGRADECIMIENTOS}

Al Instituto Oceanográfico de Venezuela de la Universidad de Oriente por el apoyo prestado para la realización de esta investigación. A María Elena Amaro, Luis Ariza, Adriana López, Marlin Medina, Lisette Molins y José L. Prin por la colaboración prestada durante el trabajo de campo y laboratorio. Igualmente, los autores desean agradecer a evaluadores anónimos que realizaron aportes interesantes en la elaboración del artículo.

\section{RESUMEN}

Con la finalidad de evaluar la asociación de índices de crecimiento en estadios tempranos de peces marinos, se estimó la tasa de crecimiento somático y las condiciones fisiológicas de Eucinostomus argenteus en dos zonas del nor-oriente venezolano: Bahía de Mochima y Golfo de Cariaco. La edad y el crecimiento fueron estimados basados en análisis de otolitos sagitta. Las condiciones fisiológicas fueron evaluadas por medio de las concentraciones de proteínas y la relación ARN/ADN, empleando técnicas espectofotométricas y fluorométricas sobre tejido muscular. Las relaciones entre tallas con la edad y el diámetro de los otolitos resultaron positivas, significativas y ajustadas a un modelo de regresión lineal. Los valores de la tasa de crecimiento reciente oscilaron entre 0.178 y $0.418 \mathrm{~mm} \mathrm{día}^{-1}$, la tasa de crecimiento retrocalculado varió entre 0.295 y $0.393 \mathrm{~mm} \mathrm{día}^{-1}$, y la tasa ARN/ADN osciló entre 1.65 y 6.97 . No se registraron diferencias entre las zonas de estudio, sin embargo se reportaron diferencias entre localidades. A pesar de no encontrarse correlación entre la tasa de crecimiento y la relación ARN/ADN, los valores reportados sugieren crecimiento positivo de los individuos silvestres en las localidades evaluadas. No obstante, ciertas localidades mostraron valores que indican pobres condiciones nutricionales, pudiendo afectarse a futuro otras tasas vitales.

Palabras claves: Gerreidae, tasa de crecimiento, condición nutricional, otolitos, ARN/ADN.

\section{REFERENCIAS}

Alfaro, R., C. González \& L. Martínez. 2002. Ácidos nucléicos para evaluar la condición de larvas de peces. Ciencia UANL. 2: 211-217.

Balza, M.A \& B. Marín 2000. Verificación de la marca de eclosión en los otolitos sagitales de larvas de Sardinella aurita (Pisces: Clupeidae). Rev. Biol. Trop. 48: 183-186. 
Balza, M.A., M. Lemus \& B. Marín. 2006. Crecimiento somático y relación ARN/ADN en juveniles de la sardina Sardinella aurita Valenciennes, 1847 (Pisces: Clupeidae) del Golfo de Santa Fe, Venezuela. Bol. Inst. Oceanogr. Venezuela, Univ. Oriente. 45: 41-49.

Balza, M.A., M. Lemus \& B. Marín. 2007. Tasa de crecimiento en larvas de Sardinella aurita Valenciennes, 1847 (Pisces: Clupeidae) del Morro de Puerto Santo, Venezuela. Interciencia. 32: 333-338.

Bergeron, J.P. 1997. Nucleic acids in ichthyoplancton ecology: a review, with emphasis on recent advances for new perspectives. J. Fish. Biol. 51: 284-302.

Bradford, M. 1976. A rapid and sensitive method for the quantization of microgram quantities of protein utilizing the principle of protein-dye binding. Anal. Biochem.72: 248-254.

Brothers, E.D. \& W.N. McFarland. 1981. Correlations between otolith microstructure, growth, and life history transitions in newly recruited french grunts (Наетulon flavolineatum (Desmarest), Haemulidae). Rapp. P.-v. Réun. Cons. Int. Explor. Mer. 178: 369-374.

Brothers, E., D. Williams \& P. Sale. 1983. Length of larval life in twelve families of fishes at One Tree Lagoon, Great Barrier Reef, Australia. Mar. Biol. 76: 319-324.

Buckley, L. 1984. RNA - DNA ratio: an index of larval fish growth in the sea. Mar. Biol. 80: 291-298.

Bulow, F. 1987. RNA-DNA ratios as indicators of growth in fish: A review, p. 45-64. In R.C. Summerfelt \& G.E. Hall (eds.). The age and growth of fish. Iowa State University, Iowa.

Caldarone, E. 2005. Estimating growth in haddock larvae Melanogrammus aeglefinus from RNA:DNA ratios and water temperature. Mar. Ecol. Prog. Ser. 293: 241-252.

Caldarone, E., C. Clemmesen, E. Berdalet, T. Miller, A. Folkvord, G. Holt, M. Olivar \& I. Suthers. 2006. Intercalibration of four spectrofluorometric protocols for measuring RNA/DNA ratios in larval and juvenile fish. Limnol. Oceanogr. Methods. 4: 153-163.

Campana, S. 1992. Measurement and interpretation of the microstructure of fish otoliths. 59-71 p. In D. K. Stevenson \& S. E. Campana (eds). Otolith microstructure examination and analysis. Can. Spec. Publ. Fish. Aquat. Sci. 117.

Campana, S. \& S. Thorrold. 2001. Otoliths, increments, and elements: keys to a comprehensive understanding of fish populations? Can. J. Fish. Aquat. Sci. 58: 30-38.
Cervigón, F. 1993. Los peces marinos de Venezuela. Volumen II. Fundación Científica Los Roques, Venezuela, $498 \mathrm{p}$.

Claro, R. \& K. Lindeman. 2003. Spawning aggregation sites of snapper and grouper species (Lutjanidae and Serranidae)on the insular shelfof Cuba. Proc. Gulf. Carib. Fish Ins. 14: 91-106.

Clarke, K.R. 1993. Non-parametric multivariate analysis of changes in community structure. Aust. J. Ecol. 18: 117-143.

Clemmesen, C. 1993. Improvements in the fluorometric determination of the RNA and DNA content of individual marine fish larvae. Mar. Ecol. Prog. Ser. 100: 177-183.

Clemmesen, C. \& T. Doan. 1996. Does otolith structure reflect the nutritional condition of a fish larvae? Comparison of otolith structure and biochemical index (RNA/DNA ratio) determined on cod larvae. Mar. Ecol. Prog. Ser. 138: 33-39.

Cunha, I., F. Saborido-Rey \& M. Planas. 2003. Use of multivariate analysis to asses the nutritional condition of fish larvae from nucleic acids and protein content. Biol. Bull. 204: 339-349.

Expósito, N. 1997. Estudio de los efectos de las descargas de una Laguna de oxidación sobre las comunidades planctónicas en la Bahía de Mochima (Edo. Sucre). Trabajo Especial de Grado, Escuela de Biología. Universidad Central de Venezuela, Caracas, Venezuela. $154 \mathrm{pp}$.

Fonseca, V., A. Serafim, R. Company, M. Bebianno \& H. Cabral. 2009. Effect of Cooper exposure on growth, condition índices and biomarker response in juveniles ole Solea senegalensis. Sci. Mar. 73: 51-58.

Frommel, A. \& C. Clemmesen. 2009.Use of biochemical indices for analysis of growth in juvenile two-spotted gobies (Gobiusculus flavescens) of the Baltic Sea. Sci. Mar. 73S1: 159-170.

Gibson, R. 1994. Impact ofhabitat quality and quantity on the recruitment of juvenile flatfishes. Neth. J. Sea Res. 32: 191-206.

Gilliers, C., O. Le Pape, Y. Désaunay, J.P. Bergeron, N. Schreiber, D. Gueralut \& R. Amar. 2006. Growth and condition of juvenile sole (Solea solea L.) as indicators of habitat quality in coastal and estuarine nurseries in the Bay of Biscay with a focus on sites exposed to the Erika oil spill. Sci. Mar. 70S1: 183-192. 
Grandcourt, E.M., T. Al Abdessalaam, F. Francis \& T. Al Shamsi. 2006. Fisheries biology of a short-lived tropical species: Gerres longirostris (Lacépéde, 1801) in the Arabian Gulf. ICES J. Mar. Sci. 63: 452-459.

Houde, E. 2009. Recruitment Variability. 91-171. In T. Jakobsen. M. Fogarty, B. Megrey \& E. Mokness (eds.). Fish reproductive biology and its implications for assessment and management. Blackwell Science Ltd, Oxford, U.K.

Jones, C. 2002. Age and growth. p. 33-63 In L. Fuiman \& R. Werner (ed.) Fishery Science: The unique contributions of early life stages. Blackwell Scienc.

Leslie, I. 1955. The nucleic acid content of tissues and cells. p. 1-50. In The nucleic acids, chemistry and biology. E. Chargaff \& J. Davidson (ed.). Academic Press, Inc., Nueva York. USA

Marín, B., C. Lodeiros, D. Figueroa \& B. Márquez. 2004. Distribución vertical y abundancias estacional del microzooplancton y su relación con los factores ambientales en Turpialito, Golfo de Cariaco, Venezuela. Revista Científica, FCV-LUZ. XIV: 133-139.

Marín, B. 2009. Estudios en comunidades ictioplanctónicas del Golfo de Cariaco. Trabajo de Ascenso. Dept. Biología, Univ. Oriente, Cumaná, Venezuela. 82 p.

Martín, D., E. González \& D. Hernández.1995. Prioridades de Conservación de las áreas marinos-costeras de Venezuela Fudena.W W F. Caracas.

Márquez, B., B. Marín, J.R. Díaz-Ramos, L. Troccoli \& S. Subero-Pino. 2007. Variación estacional y vertical de la biomasa del macrozooplancton en la Bahía de Mochima, Estado Sucre-Venezuela, durante 19971998. Revista de Biología Marina y Oceanografía. 42: $241-252$

Meekan, M., J. Carleton, A. McKinnon, K. Flynn \& M. Furnas. 2003. What determines the growth of tropical reef fish larvae in the plankton: food or temperature? Mar. Ecol. Prog. Ser. 256: 193-204.

Miloslavich, P., E. Klein, E. Yerena \& A. Martín. 2003. Marine biodiversity in Venezuela: Status and Perspectives. Gayana. 67: 275-301.

Montaño, O.2009. Catch dynamics, growth, and reproduction of striped mojarra Eugerres plumier in Lake Maracaibo, Venezuela. Ciencia. 17: 141-150.

Narváez, M. 2011. Variación temporal y especial del micro y mesozooplancton en la parte interna de la Bahía de Mochima, Estado Sucre, Venezuela, en época de surgencia. Trabajo Especial de Grado, Dept. Biología, Univ. Oriente, Cumaná, Venezuela. 64 pp.

Neuman, M., D. Wittin \& K. Able. 2001. Relationships between otolith microstructure, otolith growth, somatic growth and ontogenetic transitions in two cohor ts of windowpane. J. Fish. Biol. 58: 967-984.

Okuda, T., A. Benítez, J. García \& F. Fernández. 1968. Condiciones hidrográficas y químicas en la Bahía de Mochima y la Laguna Grande del Obispo, desde 1964 a 1966. Bol. Inst. Oceanogr. Univ. Oriente. 7:7-32.

Okuda, T., Benítez-Alvares, J., Bonilla, J. \& Cedeño, G. 1978. Características hidrográficas del golfo de Cariaco, Venezuela. Bol. Inst. Oceanogr. Univ. Oriente. $17: 69-88$.

Panella, G. 1971. Fish otoliths: daily growth layers and periodical patterns. Science. 173: 1124-1127.

Peck, M., A. Buckley, E. Caldarone \& D. Bengston. 2003. Effects on food consumption and temperature on growth of larval and juvenile red drum (Scianops ocellatus). Mar. Ecol. Prog. Ser. 251: 233-243.

Quintero, A., J. Bonilla, L. Serrano, M. Amaro, B. Rodríguez, G. Terejova \& Y. Figueroa. 2004. Características ambientales de la Bahía de Mochima y adyacencias de la Cuenca de Cariaco, Venezuela. Bol. Inst. Oceanogr. Venezuela, Univ. Oriente. 43: 49-64.

Ramírez, T. \& B. Marín. 2006. Edad y crecimiento en larvas de Sardinella aurita (Pisces: Clupeidae) del nororiente de Venezuela, mediante el análisis de sus otolitos. Ciencias Marinas. 32: 559-567.

Randall, J. 1967. Food habits of reef fishes of The West Indies. Stud. Trop. Oceanogr. 5: 665-847.

Raventós, N. \& E. Macpherson. 2001. Planktonic larval duration and settlementmarks on the otoliths of Mediterraneon litoral fishes. Mar. Biol. 138: 1115-1120.

Richard, P., J. Bergerson, M. Boulhic, R. Galois \& J. Leruyet. 1991. Effect of starvation on RNA-DNA and protein content of laboratory-reared larvae and juveniles of Solea solea. Mar. Ecol. Prog. Ser. 72: 69-77.

Rivas, A., E. Méndez, L. Ruiz, A. Torres \& L. Martínez. 1999. Hábitos alimenticios de Eucinostomus gula y E. argenteus (Pices: Gerreidae) en la Bahía de Mochima, Estado Sucre, Venezuela. Bol. Inst. Oceanogr. Venezuela, Univ. Oriente. 38: 91-97.

Rubio, E., J. Loaiza \& C.J. Moreno. 2004 Crecimiento y sobrevivencia de dos especies de mojarras Diapterus peruvianus y Eucinostomus currani criadas en jaulas 
flotantes en la Bahia de Buenaventura. Mem. III Congreso Iberoamericano virtual de acuacultura. CIVA 2004. Zaragoza, España.

Sadhotomo, B. 1983. The dynamics of trevally, Pentaprion longimanus at Java Sea. Mar. Fish. Res. Report, Research Report, 28: 82 pp.

Secor, D. H. \& J. M. Dean. 1989. Somatic growth effect on the otolith-fish size relationship in young pond-reared striped bass, Morone saxatilis. Can. J. Fish. Aquat. Sci. 46: 113-121.

Secor, D. H. \& J. M. Dean. 1992. Comparison of otolithbased back-calculation methods to determine individual growth histories of larval striped bass, Morone saxatilis. Can. J. Fish. Aquat. Sci. 49: 1439-1454.

Secor, D.H., J.M. Dean \& E.H. Laban. 1992. Otolith removal and preparation for microstructural examination, p. 19-57. In: D.K. Stevenson \& S.E. Campana (ed.) Otolith microstructure examination and analysis. Can. Spec. Publ. Fish Aquat. Sci. 117.

Sokal, R. \& F. Rohlf. 1995. Biometry. 3ra Edición. W.H. Freeman and Company, Nueva York. 887 pp.

Sponaugle, S., J. Llopiz, L. Havel \& T. Rankin. 2009. Spatial variation in larval growth and gut fullness in a coral reef fish. Mar. Ecol. Prog. Ser. 383: 239-249.
Sponaugle, S. 2010. Otolith microstructure reveals ecological and oceanographic processes important to ecosystem-based management. Environ. Biol. Fish. 89: 221-238.

Sponaugle, S., K. Walter, K. Denit, J.Llopiz \& R. Cowen. 2010. Variation in pelagic larval growth of Atlantic billfishes: the role ofprey composition and selective mortality. Mar. Biol. 157: 839-849.

Stevenson, D. \& S. Campana. 1992. Otolith microstructure examination and analysis. Can. Spec. Publ. Fish. Aquat. Sci. 117.

Swearer, S., J. Caselle, D. Lea \& R. Warner. 1999. Larval retention and recruitment in an island population of a coral-reef fish. Nature. 402: 799-802.

Thorrold R. \& D. Williams. 1989. Analysis of otolith microstructure to determine growth histories in larval cohorts of a tropical herring (Herklotsichthys castelnaui). Can. J. Fish. Aquat. Sci. 46: 1615-1624.

Tucker, J.W. \& D.E. Jory. 1991. Marine fish culture in the Caribbean region. World Aquac. 22: 10-27.

Wilson, D. \& M. McCormick. 1999. Microstructure of settlement marks in the otoliths of tropical reef fishes. Mar. Biol. 134: 29-41. 
